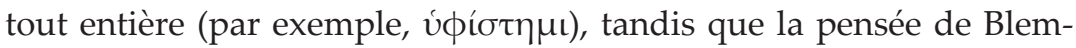
mydès semble ici plus nuancée, car il ne présuppose aucune réciprocité entre les propriétés hypostatiques. Quoi qu'il en soit, la condition

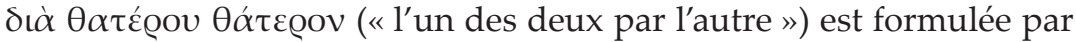
Blemmydès comme une condition stricte, ce qui s'accorde d'ailleurs avec l'ensemble de sa pensée trinitaire. Jusqu'ici, la compréhension de Blemmydès par Stavrou (et par moi-même) est cohérente. Malheureusement, elle cesse de l'être dans un contexte plus large de la tradition byzantine, car nous ne trouvons pas une pareille condition explicite chez Grégoire de Chypre, c'est-à-dire dans la version " canonique » traditionnelle de la doctrine de Blemmydès. Dans l'état actuel de nos connaissances de la théologie de Grégoire de Chypre aussi bien que de celle de ses adversaires du camp antilatin (comme, par exemple, Jean Chilas, Georges Moschambar), il serait prématuré de se prononcer d'une manière définitive, s'il agit d'une divergence stylistique (l'attitude de Stavrou ; cf., au dernier lieu, M. Stavrou, Le théologien Nicéphore Blemmydès (1197-v. 1269), figure de contradiction entre orthodoxes et latinophrones, OCP 74 (2008) 165-170) et tactique ou bien d'une vraie différence doctrinale. Le travail de Stavrou est devenu cependant un nouveau stimulus pour les recherches sur les discussions trinitaires dans le camp antilatin au dernier quart du XIII ${ }^{e}$ siècle. Je suis sûr que ces recherches jetteront de la lumière également sur l'origine des querelles hésychastes.

Cela dit, je n'ai qu'à répéter que le travail de Stavrou présente maintenant la reconstruction de la pensée de Blemmydès la plus convaincante. On attend avec impatience le tome 2.

Basile Lourié

Tedros Abreha, Il Gädl di Abuna Demyanos santo eritreo (XIV/ $X V$ sec.). Edizione del testo etiopico e traduzione italiana (Patrologia Orientalis Tome 50. Fascicule 2. $\mathrm{N}^{\circ} 223$, Turnhout, Belgique: Brepols, 2007). Pp. 125. [= 113-237]. Prezzo: $€$ 46. [Senza ISBN]

L'edizione del testo del Gadla Demyānos pubblicato con introduzione, traduzione e commento a cura di Tedros Abreha nel nuovo fascicolo della Patrologia Orientalis colma una lacuna importante nella conoscenza del corpus agiografico dei discendenti di Ëwosțātēwos, che tanto di sé hanno improntato il monachesimo diffuso sull'altopia- 
no oggi eritreo. Se ne ricava indubbiamente nuova luce e una meno incompleta conoscenza delle tradizioni sulla personalità del monaco Demyānos - immediato predecessore di Yonās nella linea genealogica che da Absādi, successore di Ēwosțātēwos, procede a Musē e via Demyānos a Yonās stesso - se è vero che non se ne aveva alcuna notizia nell'ampia documentazione eustaziana indagata da Gianfrancesco Lusini, Studi sul monachesimo eustaziano (secoli XIV-XV) (Studi Africanistici. Serie Etiopica 3), Napoli 1993, e che non gli sono oggi riconducibili chiese o monasteri. Notizie al suo riguardo già offriva una serie di testi raccolti dalla Missione Italiana in Eritrea (MIE) dell'Università di Bologna (1992 sgg.) diretta da Irma Taddia, e pubblicati nell'occasione ancora da Lusini, "Scritture documentarie etiopiche (Dabra Dehuhān e Dabra Șegē, Sarā'ē, Eritrea)", Rassegna di Studi Etiopici, 42 (1998 [1999]), pp. 5-55, ms. MIE 17, doc. I, f. $1^{\text {r-v }}$, trad. pp. 22-23, testo pp. 26.7, 26.17 (genealogia Ēwostātēwos, Absādi, Musē, Demyānos,

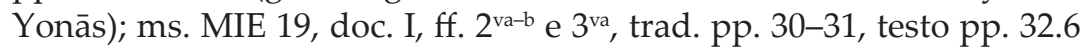
(data di nascita di Demyānos nell'anno di grazia $33=1381$ ), 32.23 (salvamento di Ṭarāqit nell'anno $80=1428$ : a tal proposito, non credo si possa concordare con Tedros, p. 126, n. 28, nel ritenere che il nome non sia un toponimo e valga, scilicet come participio femminile, "ammalata di dissenteria", perché Țarāqit è altrove chiaramente un luogo, cfr. ms. MIE 22, doc. V, f. $4^{\text {va, }}$, trad. p. 44, testo p. 47.25) e 32.36 (commemorazione di Demyānos il 28 yakkātit); MIE 22 I, f. $6^{\text {ra }}$, trad. p. 40, testo p. 46.2 (morte di Demyānos nel $7^{\circ}$ anno di regno di Zar'a Yā'qob = 1441) e MIE 22, doc. V, ff. $3^{\text {va }}$ e $4^{\text {va }}$, trad. p. 44 , testo pp. 47.7 e 47.28 (genealogia).

L'esperienza della frequentazione "sul campo" delle comunità monastiche di oggi e la diretta conoscenza dei luoghi, combinate con la rassegna puntuale della bibliografia relativa e il tentativo di spiegazione globale del testo del gadl (in particolare, con il reperimento di citazioni e allusioni alla letteratura biblica, monastica, liturgica), hanno permesso all'Editore di fornire varie rettifiche e non pochi dati nuovi (spec. pp. 124-127; e a p. 123, n. 16 si annuncia che anche l'edizione del Gadla Fiqtor è in preparazione, cfr. ora anche Tedros Abraha, "Controversie sul Sabato e sul Millennio secondo i Gädl inediti di Täwäldä-Mädəhn (sic) e di Fiqətor", in Scrinium 4 (2008) = V. BaranovB. Lourié (eds.), Patrologia Pacifica. Selected papers presented to the Western Pacific Rim Patristics Society 3rd Annual Conference (Nagoya, Japan, September 29 -October 1, 2006) and other patristic studies, Санкт-Петербург 2008, pp. 79-102). Da tradizioni orali, a quanto pare (p. 125), risulta precisamente collocabile la chiesa di Dabra Sinā, oggi dedicata a Ma- 
ria (ma in origine, unica finora nota, a Demyānos), anziché nel Daqqi Tasfā - come indicato da Lusini, "Dəmyanos", in EAE (Siegbert Uhlig (ed.), Encyclopaedia Aethiopica, Wiesbaden 2003 sgg.), vol. 2, p. 132b, sulla scorta di Carlo Conti Rossini, "Gli Atti di Abbā Yonās", in Rendiconti della Reale Accademia dei Lincei. Classe di scienze morali, storiche e filologiche, s. 5a, 12 (1903), pp. 177-201, 239-262, spec. p. 178 e n. 1, e p. 187.23-24 - nel distretto del Zāydakkolom, sempre nel Sarāya, al pari di altri luoghi legati all'attività del monaco: Endā Abbā Sem'on (pp. 162 / 163, §§ 33-34, probabilmente lo stesso citato alle pp. 138 / 139, §4, e 136, n. 25), l'oscuro Dabra Salām (cfr. p. 126, n. 25), o Dabra Dehuhān, ove risulterebbero conservati i resti di Demyānos (p. 125 e n. 23). Per il resto, mentre Dabra Sinā figura nel testo solo in una nota marginale, a glossare il generico «deserti di Bäräka» nel ms. B (pp. 160, apparato, n. 62 / 161, n. 91, § 32, non incluso nell'indice, come forse sarebbe stato opportuno almeno per alcuni termini di maggior rilievo che figurano solo nelle note o nell'apparato), il Bur con Aramo (Hagara Masqal di pp. 182, 204 / 183, 205, §§ 59, 88) e i meno perspicui (forse cuscitici) Dabla Sumā e Dalu'etā, lo Sira con Zāger, il Ḥamāsēn, costituiscono l'orizzonte geografico della formazione e della predicazione del santo.

Tutte concentrate nella parte centrale e terminale della sua vita sono invece le figure storiche evocate, tra le quali l'oscuro governatore del Sira, Dēganā (p. 127), il cui omonimo Tegrē Makwannen in Karolus Conti Rossini, Documenta ad illustrandam historiam. I. Liber Axumae, 2 voll. (Corpus Scriptorum Christianorum Orientalium s. altera, 8; rist. 54 e 58, Scriptores Aethiopici 24 e 27), Parisiis 1909-10 (rist. Louvain 19611962), doc. nr. 51 (testo p. 43.15, trad. p. 51.23), è altrimenti attestato all'epoca del re Galāwdēwos; il nebura 'ed Nob (cfr. p. 215, n. 27) - su cui cfr. ora Claire Bosc-Tiessé - Marie-Laure Derat, "Nob", in EAE, vol. 3, p. 1192 - del quale, stando al gadl, Demyānos sarebbe stato il fratello del padre, nonché hadāni, «tutore» (pp. 214-216 / 215-217, §§ 101-103); ma anche i re Yesḥaq e Hezba Nāñ (Yenāyn nel testo, pp. 210 / 211, $\S \S 96 \mathrm{sgg}$.), presso la corte dei quali il santo muore, sicché la sua salma è poi reclamata dai suoi compaesani e loro dal re concessa. Nell'insieme, il testo pare mantenersi sostanzialmente lontano dal tono e dalla tensione propria di altre agiografie eustaziane, e si dispiega nell'esaltazione delle virtù monastiche e taumaturgiche del protagonista, se pur più punti narrativi restano da chiarire: la sofferenza del santo per il forzato richiamo e la permanenza a corte potrebbero nascondere, sotto il disagio agiografico per l'impossibile rifiuto del mondo, la prigionia o la relegazione presso il re. Il testo presuppone per di più quasi certamente la redazione dell'agiografia del suo successore Yonās, dato che 
del Gadla Yonās è richiamato un episodio (pp. 208 / 209 sgg., e p. 211, n. 16, §§ 92-95, 98-99), e la mancata insistenza sull'osservanza del sabato porta la datazione del gadl a un'epoca posteriore alle controversie eustaziane stesse, come l'Editore giustamente nota (pp. 123-124). Nel complesso, pur dopo questa prima analisi di Tedros, resta ancora spazio per approfondire la natura del rapporto tra la tradizione agiografica e quella documentaria, entrambe fortunatamente presenti nel caso di Demyānos.

L'edizione del testo si fonda su due testimoni (un po' impropriamente indicati come «fonti», p. 117): il ms. A, ff. $125^{\mathrm{ra}}-184^{\mathrm{vb}}$, del XVXVI sec., direttamente visionato e nuovamente descritto con qualche elemento aggiuntivo rispetto alle prime segnalazioni, già fornite di indicazioni sulla fascicolazione, conservato presso il monastero di Dabra Dehuhān (stando alla descrizione di Tedros, il f. 3, con la nota cronografica del doc. I = f. 2 di Lusini, "Scritture", pp. 29 sgg.), cfr. Alessandro Bausi - Gianfrancesco Lusini - Irma Taddia, "Materiali di studio dal Sära'è (Eritrea): le istituzioni monastiche e la struttyura della proprietà fondiaria", in Africa (Roma), 48/3 (1993), pp. 446-463, spec. p. 462, e Lusini, "Scritture", pp. 29-32, ms. MIE 19, con pubblicazione di varie note aggiunte; e un secondo, nuovo, ms. B, pp. 174-315, probabilmente del XVIII sec., esaminato in fotocopia e conservato presso il monastero di Māryām Șeyon presso 'Addi Wusseks, non lontano da Māy Demā, Sarāya. Del ms. A è inoltre pubblicata e tradotta una finora inedita nota aggiunta al f. $2^{\mathrm{r}}$, con le date di morte di varie personalità eustaziane, tra cui ancora Demyānos il 28 di yakkātit (p. 124, n. 17).

Seguono alcune osservazioni più puntuali sul testo (testo e trascrizioni del volume sono riportati tali e quali, tra «caporali» la traduzione). - P. 118: nella descrizione dei testi vengono dati come incipit le formule iniziali che incipit non sono. - p. 119.11 sgg.: i rapporti tra $\mathrm{i}$ mss. sono definiti utilizzando elementi che appartengono alla "critica delle forme" nella sua accezione più superficiale, e che vanno piuttosto inquadrati nella prospettiva delle varianti linguistico-grafiche; il ms. B (XVIII sec.) non può essere «l'antigrafo» di A (XV/XVI sec.); ne potrebbe se mai essere "l'apografo": la questione, che è essenziale, andava però ulteriormente chiarita; in ogni caso, non possono essere tacciati di «incuranti o forse inconsapevoli ...» i copisti dei codici A e B per la mancata distinzione tra laringali e sibilanti: la confusione, come noto, è antica, né ci è nota alcuna tradizione manoscritta a noi conservata che rispetti perfettamente la grafia etimologica; l'apparato, giustamente, non registra tali «errori ortografici», mentre «l'interpunzione è stata sovente "riaggiustata" » dall'Editore, senza che il lettore abbia modo di 
sapere dove e come; cfr. a tal riguardo Alessandro Bausi - Alessandro Gori, Tradizioni orientali del «Martirio di Areta». La Prima recensione araba e la Versione etiopica. Edizione critica e traduzione. Presentazione di Paolo Marrassini, Firenze 2006 (Quaderni di Semitistica, 27), pp. 112-113. pp. 119-120: tra gli errori ricorrenti propri del testo, è segnalato (p. 120) (DTP' : infinito flesso secondo la forma I.2, mentre Tedros ne ammette unicamente la forma I.1 e non esita a correggere in tal senso (cfr.

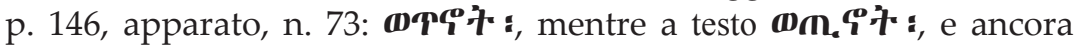

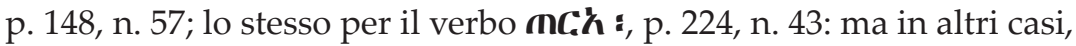
tale "rigore" necessario manca: cfr.p. 206, n. 8: «hayäsä» recte "hayyasa"):

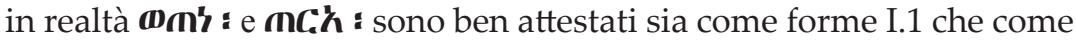
I.2, tanto che entrambe sono registrate in August Dillmann, Lexicon linguae Aethiopicae, Lipsiae 1865, cc. 941, 1220: non è quindi «uno dei casi di forme lessicali chiaramente errate»: e stupisce leggere che le forme attestate dai testimoni manoscritti sono state corrette quando «sono erronee», il che avrebbe senso nella prospettiva di un metodo consapevolmente praticato, ovviamente, «o per lo meno superate», il che non lo ha affatto: in tal modo, quell'embrione di diacronia che potrebbe cogliersi nei testi etiopici viene schiacciato sulla norma dell'insegnamento tradizionale di oggi, di cui Tedros un po' troppo si compiace, cfr. «"all'esercito" (i.e. alla coniugazione)» (p. 120), e varie altre volte nelle annotazioni; ciò disturba tanto più che per altri aspetti l'Editore mette in luce l'importanza, non solo dell'individuazione delle citazioni e allusioni a testi biblici ed extra-biblici (in questo lo sforzo di Tedros è veramente ammirevole), ma anche della sopravvivenza in esse di fasi testuali arcaiche del testo biblico (cfr. p. 128; p. 136, n. 20; p. 141, n. 35); il principio è ribadito a p. 127: «si è cercato di sostituire alcune forme gə'əz sorpassate», in pieno conflitto con lo scopo prefisso «di recuperare il testo originale», ibid. - p. 120: interessante l'osservazione

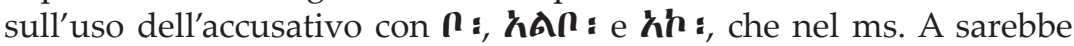
arbitrario per arcaismo. - pp. 121-123: l'Editore sottolinea l'importanza del "modello scritturale" (i riferimenti al quale, tenuto conto della precisione e profondità che alcuni contributi di agiografica etiopica hanno raggiunto al riguardo, sono un po' generici), e della tessitura di allusioni e citazioni bibliche e letterarie; sembra però fraintendere che la dichiarazione dello scrittore («povero di intelligenza e dalla parola stentata») sulla propria inadeguatezza obbedisce a un ben noto tópos. -

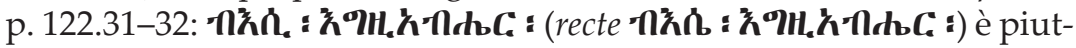

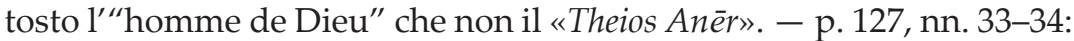
«Lusini 1988»: “Lusini 1998". - pp. 134.2 / 135.3: 7-nl: / «creò»:

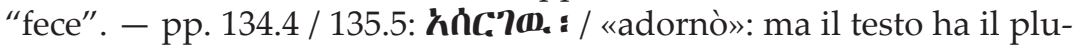


rale. - pp. 134.13 / 135.11: $\boldsymbol{P} \boldsymbol{d} \boldsymbol{W} \cdot \boldsymbol{C}: \mathbf{~}$ / «si trascina»: "cammina, procede". - pp. 134.16 / 135.13-14: "prostrandosi". - p. 136.16: "iT", : recte "1T" : - p. 137, n. 14: la Vita Antonii è data come certamente tradotta dal greco, ma il testo è stato finora oggetto di indagini solo esplorative. - pp. 138.22 / 139.30:

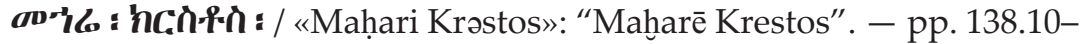
11 / 139.15: H†w, pecore del gregge»: "fu messo a capo" ecc. - pp. 138.27 / 139.36:

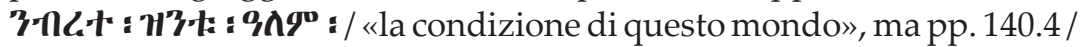
141.4-5: id. / «il soggiorno in questo mondo». - pp. 140.1 sgg. / 141.3 sgg.: i tempi della trad. sono incoerenti. - pp. 140.3 / 141.5: P-hA P*: /

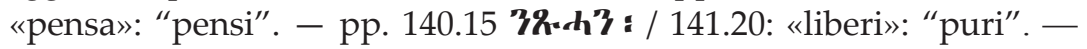

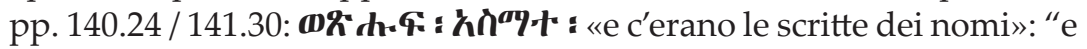

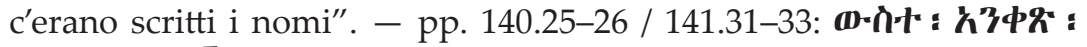

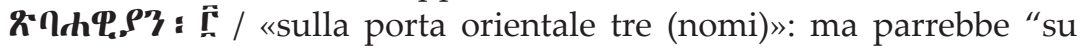
una porta i tre orientali" ecc., anche se l'espressione resta oscura. -

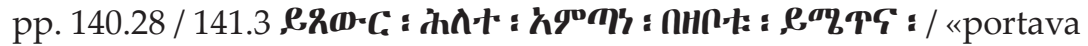
la verga della misura per misurarvi»: "portava una verga della misura con cui misurare", con frase relativa restrittiva. - pp. 140.29 / 141.36:

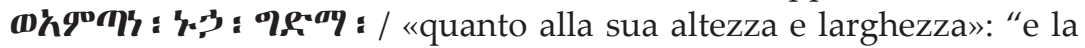
sua lunghezza è pari alla sua larghezza". - pp. 140.30 / 141.37:

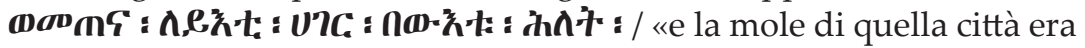
di tali proporzioni»: "e la misura di quella città era di quella verga". -

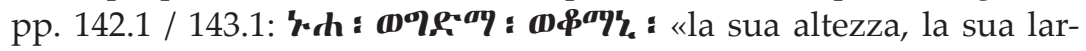
ghezza e la sua statura»: "la sua lunghezza, la sua larghezza e la sua

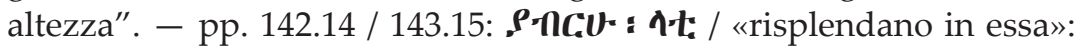

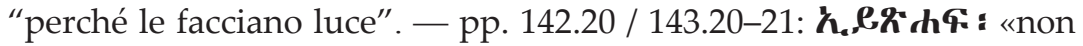

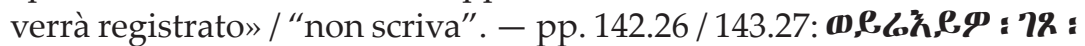

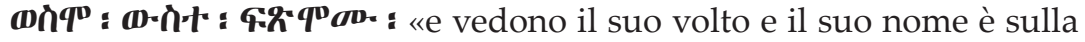
loro fronte»: "e vedono il suo volto e il suo nome sulla loro fronte". -

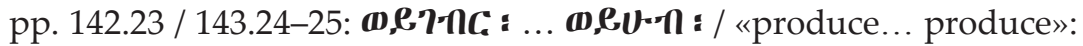
"produce... fornisce". - pp. 142.29 / 143.32: †n். : / «ripose»: "aven-

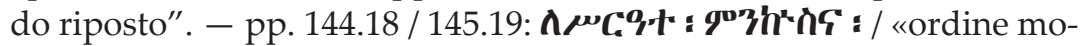

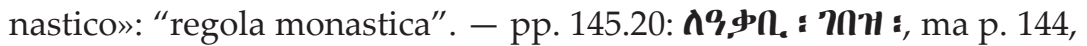

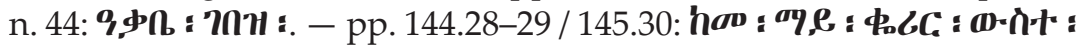

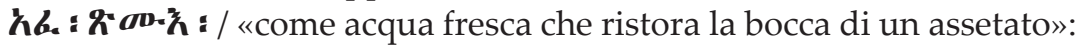

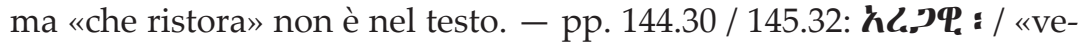
gliardo»: potrebbe trattarsi di una allusione allo Aragāwi Manfasāwi. -

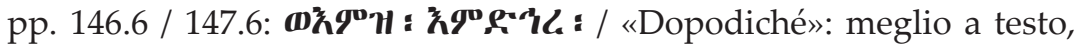

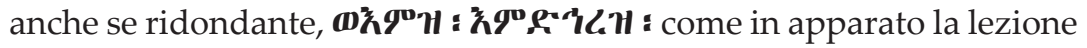


del ms. A. - pp. 146.23 / 147.26: (oח+1990 \& : / «nel digiuno»: "nella

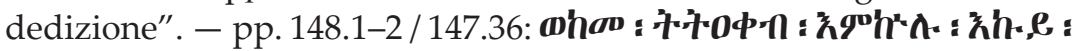

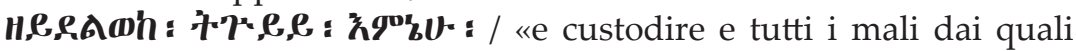
dovrai fuggire»: "e che tu ti guardi da tutti i mali da cui ti conviene fuggire". - pp. 148.3 / 149.2: H, serà". - pp. 148.18 / 149.22: , $\mathbf{G} \mathbf{6} \mathbf{2 9 0}$ : / «vi riporti alla perfezione»: "vi riporterà alla perfezione". - pp. 152.8 / 153.13: CC:

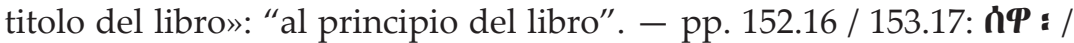
«birra»: cfr. p. 153, n. 67: l'etimologia di sawā, "birra" da șewā', "calice", è assolutamente inaccettabile; il termine śawā, poi, è attestato in etiopico con il significato di "birra" sin dai testi epigrafici aksumiti, cfr. le celebri occorrenze in DAE (Enno Littmann, Deutsche-Aksum Expedition herausgegeben von der Generalverwaltung der königlichen Museen zu Berlin. Band IV. Sabäische, griechische, und altabessinische Inschriften, Berlin 1913), nrr. 6.11, 7.13 = RIÉ (Étienne Bernand - Abraham Johannes Drewes - Roger Schneider, Recueil des Inscriptions de l'Éthiopie des périodes pré-axoumite et axoumite, 3 vols. [Académie des Inscriptions et Belles-Lettres], Paris 1991-2000), vol. I, nrr. 185 I.11, II.13, e le parallele RIÉ nrr. 185 bis I.13, II.(C).1. - pp. 154.25 / 155.31 e 154, n. 75: \&.A : / «campana»: la forma è segnalata in ulteriori testi, cfr. Alessandro Bausi, "Su alcuni manoscritti presso comunità monastiche dell'Eritrea [III]", in Rassegna di Studi Etiopici, 41 (1997 [1998]), pp. 13-56, spec.

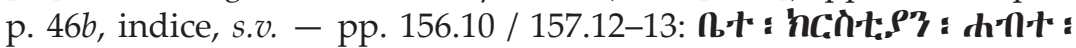

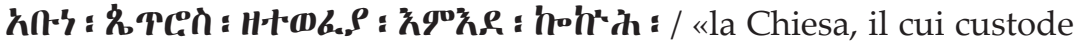
è il nostro Padre Pietro che l'ha ricevuta dalla mano della Roccia»: ma

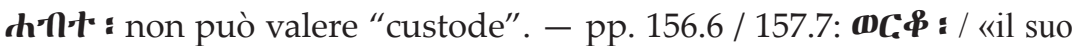

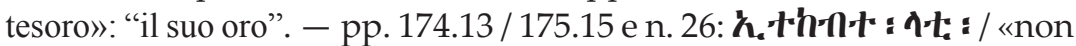

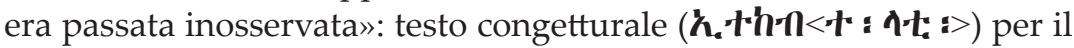

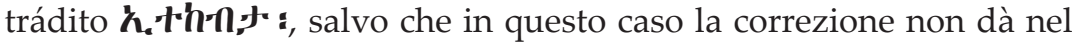

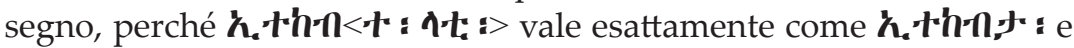
qui sarà forse da pensare a costruzione a senso o a interferenza con forma di gerundio tigrino (forme e parole anomale, amariche, ma soprattutto tigrine e tigrineggianti sono segnalate nelle note a più ripre-

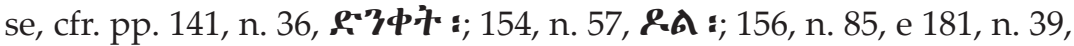

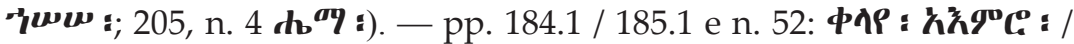
«mare di conoscenza»: "abisso di conoscenza". - p. 202, n. 98: l'usus scribendi è evocato un po' a sproposito: nella fattispecie, l'espressione

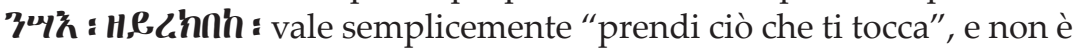
affatto «un errore», né di norma usus scribendi - contrariamente all'accezione che l'Editore fa propria - denota espressione irrazionale o 
grammaticalmente anomala: usus scribendi indica semplicemente l'uso linguistico e stilistico di un autore, di un genere, di un'epoca ecc. quale che esso sia, e vale come criterio interno in critica testuale. - p. 212, n. 24: «Abba Mälkä Ședeq 2002» non pare reperibile in bibliografia. -

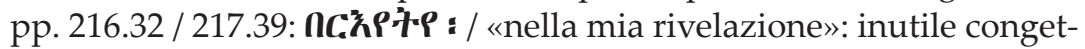

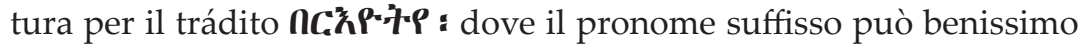
valere come oggetto: "Gioisci o mio servo Demyānos, per il fatto di vedere me". - p. 228.6: l'occorrenza di r|6.F", "pergamena", si aggiunge alle non molte sinora note a corrobare l'etimologia del termine da $\mu \varepsilon \mu \beta \mathrm{O} \alpha ́ v \alpha$, cfr. Alessandro Bausi, "La tradizione scrittoria etiopica", in Segno \& Testo, 6 (2008), pp. 507-557, spec. p. 522; per la tradizione scrittoria è ugualmente utile la menzione a p. 144.21 della $\mathbf{n}+\mathbf{*}$

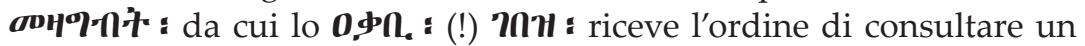

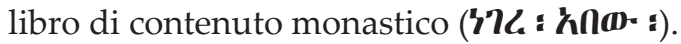

Alessandro Bausi

\section{NOUVELLES PUBLICATIONS \\ DE L'INSTITUT DE LA LITTÉRATURE RUSSE (MAISON DE POUCHKINE) SUR LA LITTÉRATURE ANCIENNE DE LA RUSSIE}

1. TODRL, tome LVIII (2007). Tрудъ Oтдела древнерусской литературь. Том LVIII (Санкт-Петербург: Наука, 2007) 964 c.

Travaux du Secteur de la littérature russe ancienne. Tome LVIII (St Pétersbourg: Nauka, 2007) 964 p. ISBN 978-5-02-026502-8. [Hommage à Gelian Mikhailovitch Prokhorov]

2. Г. М. Прохоров, «Так воссияют праведники...» Византийская хитература XIV в. в Древней Руси (Санкт-Петербург: Изд-во Олега Абышко, 2009) (с2008) (Бибдиотека христианской мысли. Исследования) 268 с.

G. M. Рвокновоv, Alors les justes resplendiront... La littérature byzantine du XIV siècle dans la Russie ancienne (St Pétersbourg : chez Oleg Abychko, 2009) (c2008) (Bibliothèque de pensée chrétienne. Recherches) 268 p. ISBN 978-5-903525-18-8.

Le tome LVIII de la célèbre série TODRL est paru commel'Hommage à Gelian Mikhailovitch Prokhorov pour son 70 anniversaire. Un vol- 